\title{
Tracking the Orientation and Axes Lengths of an Elliptical Extended Object
}

\author{
Shishan Yang and Marcus Baum
}

\begin{abstract}
Extended object tracking considers the simultaneous estimation of the kinematic state and the shape parameters of a moving object based on a varying number of noisy detections. A main challenge in extended object tracking is the nonlinearity and high-dimensionality of the estimation problem. This work presents compact closed-form expressions for a recursive Kalman filter that explicitly estimates the orientation and axes lengths of an extended object based on detections that are scattered over the object surface (according to a Gaussian distribution). Existing approaches are either based on Monte Carlo approximations or do not allow for explicitly maintaining all ellipse parameters. The performance of the novel approach is demonstrated with respect to the state-of-the-art by means of simulations.
\end{abstract}

Index Terms-Target tracking, extended object tracking, multiplicative error, Kalman filter

\section{INTRODUCTION}

The objective of extended object tracking is to simultaneously determine both the kinematic state and the shape parameters of a moving object. With the development of novel nearfield and high-resolution sensors, extended object tracking is becoming increasingly important in many applications such as autonomous driving [1], [2] and maritime surveillance [3]. Recent overviews of extended object tracking methods and applications are given in [4] and [5].

Most sensors for extended object tracking, e.g., LiDAR or radar devices, provide a varying number of spatially distributed detections (measurements) per scan from the object. Depending on the specific sensor and target, different scattering patterns can be distinguished. For example, in two-dimensional space, measurements can be scattered on the surface of the object, or on the boundary of the object.

In case of spatially dense measurements, it might be possible to extract detailed shape information from the object. For example, star-convex shape approximations as in [6], [7], [8], [9], [10] are widely-used for this purpose. In scenarios with high measurement noise and a relatively low number of measurements from the object, it is common to approximate the object shape with an ellipse (see Fig. 1). The random matrix approaches [11], [12], [13], [14] pioneered by Koch [11] can be seen as the state-of-the-art for estimating elliptic shape approximations in case of surface scattering. By means of representing the shape estimate and its uncertainty with an Inverse-Wishart density, it is possible to derive compact closed-form expression for a Bayesian measurement update.

S. Yang and M. Baum are with the Institute of Computer Science, University of Goettingen, Goettingen 37077, Germany (e-mail: shishan.yang@cs.unigoettingen.de; marcus.baum@cs.uni-goettingen.de).

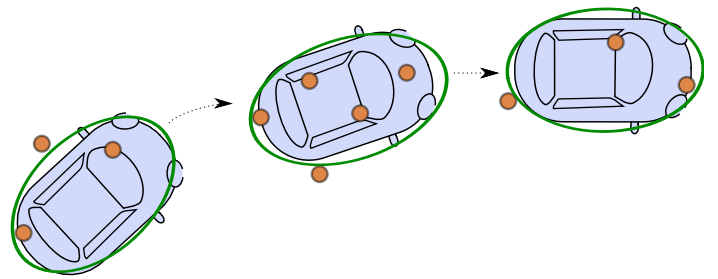

Fig. 1: Illustration of the extended object tracking problem. Multiple spatially distributed measurements are received from the target object and the objective is to determine an elliptic shape approximation in addition to the kinematic target state.

The Inverse Wishart density is defined on symmetric positivedefinite (SPD) matrices, where it is specified by

- a $d \times d$ SPD scale matrix $\mathbf{V}$ and the

- scalar degree of freedom $v \in \mathbb{R}$.

For $d=2$, the SPD scale matrix $\mathbf{V}$ can be interpreted as a twodimensional elliptic shape estimate with an uncertainty that is specified by the scalar $v$. An advantage of this representation is that the ellipse shape (including orientation, and semi-axes lengths) is uniquely defined by a single scale matrix. However, the uncertainty of the complete ellipse shape is encoded in a single one-dimensional value $v$. For this reason, it is not possible to distinguish explicitly between the uncertainty of the semi-axes and the orientation, which is often necessary in practical applications.

\section{A. Contribution}

The main contribution of this work is a novel elliptic shape tracking method that explicitly maintains an

- estimate for the orientation and semi-axes lengths, i.e., a three-dimensional vector, and

- the $3 \times 3$ joint covariance of the shape estimate.

By this means, it becomes possible to explicitly model the temporal evolution of individual shape parameters and their interdependencies, which is highly relevant for numerous practical applications. For example, it can be directly modeled that the semi-axes are fixed (and unknown) but the orientation varies.

We derive compact closed-form expressions for a recursive update of the kinematic state and the aforementioned shape parameters plus the respective covariance matrices. Due to the high degree of nonlinearity of the problem, a naïve application of standard estimation techniques such as the Extended Kalman Filter (EKF), Unscented Kalman Filter (UKF) or Second-Order Extended Kalman Filter (SOEKF) [15], [16] 
result in unsatisfactory estimation results. In fact, we have even shown in our previous work [17] that the Linear Minimum Mean Squared Estimator (LMMSE) is already for the special case of axis-aligned ellipses inconsistent.

The key components that lead to the compact closed-form expressions are the followings:

C1 An explicit measurement equation (corrupted by multiplicative noise) is formed that relates a measurement to the kinematic state and shape parameters

C2 The kinematic state and the shape parameters are decoupled, i.e., treated independently (as in the random matrix approach)

C3 The kinematic state estimate is updated using the actual measurement. However, the shape parameters are updated with a pseudo-measurement constructed from the actual measurement

C4 As the measurement equation for the kinematic parameters involves multiplicative noise, a linearization is performed for the kinematic parameters, but the multiplicative noise is kept as a random variable for the moment calculation.

C5 A standard linearization of the measurement equation for the shape parameters does not yield a feasible estimator due to the high nonlinearities. For this reason, we derive a problem-tailored second-order approximation. In order to avoid the complex calculation of Hessian matrices, we exploit that the first two moments of the pseudomeasurement can be directly derived from the covariance matrix of the actual measurement.

This article is based on the two conference papers [18], [19]. Early ideas about the use of a multiplicative noise term to model a spatial distribution were discussed in [17]. In [18], we introduce a variant of the Second Order Extended Kalman filter (SOEKF) for estimating the orientation and semi-axes lengths of an ellipse. Unfortunately, it involves complex calculations of several Hessian matrices. In [19], we develop a method that works completely without Hessian matrices. To track an unknown number of extended objects, an implementation that combines [19] and Probability Density Hypotheses (PHD) filter is presented in [20], [21]. The method introduced in this work improves over [19] by a more precise approximation of the covariance of the predicted measurement. Furthermore, a much more detailed evaluation and comparison is provided.

The shape modeling with multiplicative noise in (C1) is called Multiplicative Error Model (MEM). The approximations (C1)-(C5) are the key to a Kalman filter-based update. For this reason, the new method is called $M E M-E K F^{*}$. EKF stands for Extended Kalman Filter (EKF) and the "*" emphasizes that (C1)-(C5) are problem tailored linearization techniques and moment approximations.

\section{B. Related Work}

Related work exists in the context of random matrix approaches, random hypersurface models, and particle filtering, and optimization-based approaches.

In [22], an alternative prediction for the random matrix approach is derived that allows for kinematic state dependent predictions. The model from Lan et al. [23] can capture orientation changes using a rotation matrix and and isotropic scaling. However, both methods [22], [23] still work with Inverse Wishart densities, i.e., do not allow for explicitly maintaining the uncertainty of individual shape parameters. The work [24] assumes the principal components of the measurements to be Gaussian and [25] independently estimates the orientation and semi-axes lengths based on fitting ideas, i.e., both methods [24], [25] are not based on the common spatial distribution model.

The random hypersurface approach [8], [26] also allows to estimate elliptical shapes. However, the method discussed in [8], [26] uses the Cholesky decomposition of the shape matrix as a state vector, which has no intuitive meaning. Furthermore, the standard update in the random hypersurface approach requires a point estimate for the angle from the center to the measurement source, which is a poor approximation in case of high measurement noise. The roots of the proposed method here lie in the random hyperface approach, however, while the original random hypersurface approach uses a onedimensional scaling factor, we here use a two-dimensional scaling.

A recent overview of particle filter methods for extended object and group tracking is given in [5]. Based on RaoBlackwellization, the random matrix approach has been combined with particle filtering techniques [27]. In [28], a convolution particle filter is developed for tracking elliptical shaped extended objects. Based on a hierarchical point process and particle-based approximations, multiple elliptic targets are tracked in [29] within the PHD filter framework. Particle-based methods are also widely-used for group object tracking, e.g., [30] considers a virtual leader model for groups and a Markov Chain Monte Carlo (MCMC) method for approximating the posterior density.

A further class of methods optimizes over an entire batch of measurements in order to determine an estimate for the target object and its shape. In [31], the Probabilistic MultiHypothesis Tracking (PMHT) in combination with the random matrix model is used to track multiple extended targets. In [7], a PMHT approach for estimating star-convex shape approximations is developed. In [32], a problem-tailored probability density function is introduced in order to determine the maximum likelihood estimate of elliptical target objects.

\section{Structure}

This article is structured as follows: The next section introduces the basic models that are used for tracking an elliptical shape approximation of a single extended object. The following Section III derives the compact closed-form expressions for a recursive measurement and time update. A detailed evaluation of the proposed method is provided in Section IV Subsequently, this article is concluded in Section V

\section{Modeling an Elliptical Extended OBJect}

This section introduces the shape parameterization, measurement model, and process model for a single extended object whose shape is approximated as an ellipse. 


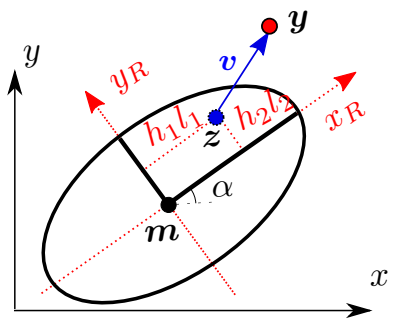

Fig. 2: An illustration of our parameterization and measurement model. We omit the time index $k$ and measurement index $i$ in this figure. The location of the object is $\boldsymbol{m}=\left[m_{1}, m_{2}\right]^{T}$. The object shape is denoted as $\boldsymbol{p}=\left[\alpha, l_{1}, l_{2}\right]^{T}$. The measurement $\boldsymbol{y}$ is measurement source $\boldsymbol{z}$ corrupted with measurement noise $\boldsymbol{v}$. The measurement source $\boldsymbol{z}$ is related to $\boldsymbol{p}$ using multiplicative noise $\boldsymbol{h}=\left[h_{1}, h_{2}\right]^{T}$. By anticlockwise rotating coordinates system $x-y$ through an angle of $\alpha$, we have the depicted ellipse axes-aligned in reference frame $x_{R}-y_{R}$.

\section{A. Parameterization}

The kinematic state of the object at time step $k$

$$
\boldsymbol{r}_{k}=\left[\boldsymbol{m}_{k}^{T}, \dot{\boldsymbol{m}}_{k}^{T}, \ldots\right]^{T}
$$

consists of the center $\boldsymbol{m}_{k} \in \mathbb{R}^{2}$, velocity $\dot{\boldsymbol{m}}_{k}$, and possible further quantities such as acceleration. As motivated in the introduction, the elliptical shape parameters at time $k$

$$
\boldsymbol{p}_{k}=\left[\alpha_{k}, l_{k, 1}, l_{k, 2}\right]^{T} \in \mathbb{R}^{3},
$$

contains the

- angle $\alpha_{k}$, which indicates the counterclockwise angle of rotation from the $x$-axis, and the

- semi-axes lengths $l_{k, 1}$ and $l_{k, 2}$.

Although this ellipse parameterization is not unique, it is quite common in tracking applications (for other measurement models), see for example [33]. The parameterization of the object extent as an SPD matrix $\mathbf{V}$ in the random matrix approaches is unique. However, the scalar parameter $v$ for the uncertainty does not allow to distinguish between the uncertainties of the orientation and the semi-axes lengths. A conversion from a covariance matrix of $\boldsymbol{p}_{k}$ to scalar $v$ induces a loss of information.

\section{B. Measurement Model}

We adopt the widely-used spatial distribution model [34], [35] for modeling the object extent. The extended object gives rise to a varying number of independent two-dimensional Cartesian detections

$$
\mathcal{Y}_{k}=\left\{\boldsymbol{y}_{k}^{(i)}\right\}_{i=1}^{n_{k}}
$$

in each time step $k$. Each individual measurement (detection) $\boldsymbol{y}_{k}^{(i)}$ originates from a measurement source $\boldsymbol{z}_{k}^{(i)}$, which is corrupted by an additive Gaussian measurement noise $\boldsymbol{v}_{k}^{(i)}$ with covariance of $\mathbf{C}^{v}$.

Each measurement source $\boldsymbol{z}_{k}^{(i)}$ lies on the object extent and follows a (uniform) spatial distribution. Similar to the random matrix approach (e.g., [12]), we approximate the uniform spatial distribution with a Gaussian spatial distribution.

A key step to the proposed method - see (C1) in the introduction - is the formulation of an explicit measurement equation, which relates a measurement source and the object state with the help of a multiplicative error term $\boldsymbol{h}$. Consider an axis-aligned ellipse that lies in the origin and its semi-axes lengths are $l_{1}$ and $l_{2}$. Any point $\boldsymbol{z}^{(i)}$ that lies on the ellipse can be written as

$$
\boldsymbol{z}^{(i)}=\left[\begin{array}{ll}
l_{1} & 0 \\
0 & l_{2}
\end{array}\right] \underbrace{\left[\begin{array}{c}
h_{1}^{(i)} \\
h_{2}^{(i)}
\end{array}\right]}_{:=\boldsymbol{h}^{(i)}} .
$$

To describe elliptical distributed measurement sources, we assume that the random variable $\boldsymbol{h}^{(i)}$ is zero mean and distributed on a unit circle. Note that if $h_{1}^{(i)}$ and $h_{2}^{(i)}$ would be independent and uniformly distributed on the interval $[-1,1]$, we would model a rectangular spatial distribution with length $2 \cdot l_{1}$ and width $2 \cdot l_{2}$.

For an ellipse with orientation $\alpha$ and center $\boldsymbol{m}$ (see Fig. 2), a rotation and translation transformation of $(3)$ gives us

$$
\boldsymbol{z}^{(i)}=\boldsymbol{m}+\underbrace{\left[\begin{array}{cc}
\cos \alpha & -\sin \alpha \\
\sin \alpha & \cos \alpha
\end{array}\right]\left[\begin{array}{cc}
l_{1} & 0 \\
0 & l_{2}
\end{array}\right]}_{:=\mathbf{S}}\left[\begin{array}{c}
h_{1}^{(i)} \\
h_{2}^{(i)}
\end{array}\right],
$$

where $\mathbf{S}$ specifies the orientation and size of the extended object. Incorporating the time index and sensor noise in (4) results in the measurement equation

$$
\boldsymbol{y}_{k}^{(i)}=\mathbf{H} \boldsymbol{r}_{k}+\mathbf{S}_{k} \boldsymbol{h}_{k}^{(i)}+\boldsymbol{v}_{k}^{(i)},
$$

where $\mathbf{H}=\left[\begin{array}{ll}\mathbf{I}_{2} & \mathbf{0}\end{array}\right]$ picks the object location out of the kinematic state.

In the same way as [12], we assume $\boldsymbol{h}_{k}^{(i)} \sim \mathcal{N}\left(\mathbf{0}, \mathbf{C}^{\boldsymbol{h}}\right)$ with

$$
\mathbf{C}^{\boldsymbol{h}}=\frac{1}{4} \mathbf{I}_{2}
$$

in order to match the covariance of an elliptical uniform distribution.

Remark 1. By assuming additive Gaussian measurement noise, the measurement likelihood becomes

$$
p\left(\boldsymbol{y}_{k}^{(i)} \mid \boldsymbol{r}_{k}, \boldsymbol{p}_{k}\right)=\mathcal{N}\left(\boldsymbol{y}_{k}^{(i)} ; \mathbf{H} \boldsymbol{r}_{k}, \mathbf{S}_{k} \mathbf{C}^{h} \mathbf{S}_{k}^{\mathrm{T}}+\mathbf{C}^{\boldsymbol{v}}\right) .
$$

We would like to note that the measurement likelihood (7) is equivalent to the likelihood used in the random matrix approach [12], which is

$$
p\left(\boldsymbol{y}_{k}^{(i)} \mid \boldsymbol{r}_{k}, \mathbf{X}_{k}\right)=\mathcal{N}\left(\boldsymbol{y}_{k}^{(i)} ; \mathbf{H} \boldsymbol{r}_{k}, z \mathbf{X}_{k}+\mathbf{C}^{\boldsymbol{v}}\right) .
$$

As $\mathbf{S}_{k} \mathbf{S}_{k}^{\mathrm{T}}$ is extension matrix $\mathbf{X}_{k}$, (7) and (8) are equivalent with $\mathbf{C}^{\boldsymbol{h}}=\frac{1}{z} \mathbf{I}_{2}$.

\section{Dynamic Model}

In general, there are no restrictions on the dynamic models for the temporal evolution of the state and shape parameters. 
For the sake of simplicity, we here assume linear equations according to

$$
\begin{aligned}
& \boldsymbol{r}_{k+1}=\mathbf{A}_{k}^{r} \boldsymbol{r}_{k}+\boldsymbol{w}_{k}^{r}, \\
& \boldsymbol{p}_{k+1}=\mathbf{A}_{k}^{p} \boldsymbol{p}_{k}+\boldsymbol{w}_{k}^{p}
\end{aligned}
$$

where

- $\mathbf{A}_{k}^{r}$ and $\mathbf{A}_{k}^{p}$ are process matrices;

- $\boldsymbol{w}_{k}^{r}$ and $\boldsymbol{w}_{k}^{p}$ are zero-mean Gaussian process noises with covariance matrices $\mathbf{C}_{r}^{\boldsymbol{w}}$ and $\mathbf{C}_{\boldsymbol{p}}^{\boldsymbol{w}}$.

\section{ESTIMATION}

This section presents closed-form expressions for the measurement and time update step based on the Kalman filter. For this purpose, we factorize the joint density for the object kinematics and extension similar to [12], [23], see also (C2) in the introduction. By this means, it is not necessary to maintain the cross-correlation between the kinematic state and the shape parameters. However, it is important to note that interdependencies between the object kinematics and extension are incorporated in the update and prediction formulas.

The derivation of a (nonlinear) Kalman filter is particularly difficult due to the high nonlinearities and zero-mean multiplicative noise in the measurement equation. To solve these issues, we derive problem-tailored approximations of the required moments by means of combining linearization and analytic moment calculation techniques.

\section{A. Measurement Update}

The measurements $\left\{\boldsymbol{y}_{k}^{(j)}\right\}_{j=1}^{n_{k}}$ from time step $k$ are incorporated sequentially in the measurement update. For this purpose, let

$$
\hat{\boldsymbol{r}}_{k}^{(i-1)}, \hat{\boldsymbol{p}}_{k}^{(i-1)} \text { and } \mathbf{C}_{k}^{\boldsymbol{r}(i-1)}, \mathbf{C}_{k}^{\boldsymbol{p}(i-1)} .
$$

denote the estimates for the kinematic state $\hat{\boldsymbol{r}}_{k}^{(i-1)}$ and shape parameters $\hat{\boldsymbol{p}}_{k}^{(i-1)}$ plus the corresponding covariance matrices, having incorporated all measurements up to time $k-1$ plus the measurements $\left\{\boldsymbol{y}_{k}^{(j)}\right\}_{j=1}^{i-1}$ from time $k$.

In the measurement update, the next measurement $\boldsymbol{y}_{k}^{(i)}$ is incorporated in order to obtain the updated estimates

$$
\hat{\boldsymbol{r}}_{k}^{(i)}, \hat{\boldsymbol{p}}_{k}^{(i)} \text { and } \mathbf{C}_{k}^{\boldsymbol{r}(i)}, \mathbf{C}_{k}^{\boldsymbol{p}(i)} .
$$

Note that - according to this notation - the predicted estimates for time $k$ are denoted as $(\bullet)_{k}^{(0)}$, correspondingly.

Remark 2. It is important to note that the measurements from a single time scan are incorporated sequentially (in an arbitrary order). Due to approximations, slightly different results might be obtained for different orderings.

As shown in [17], the object extent cannot be estimated with a linear estimator that works with the actual measurement, i.e., the shape parameters do not change when updated with a single measurement $\boldsymbol{y}_{k}^{(i)}$ in the Kalman filter framework. For this reason, a pseudo-measurement is constructed based on $\boldsymbol{y}_{k}^{(i)}$ in order to update the shape parameters. This can be seen as an uncorrelated transformation as discussed in [36].
TABLE I: Measurement update of the MEM-EKF* algorithm. Source code: https://github.com/Fusion-Goettingen/

Input: Measurements $\left\{\boldsymbol{y}_{k}^{(i)}\right\}_{i=1}^{n_{k}}$, predicted estimates $\hat{\boldsymbol{r}}_{k}^{(0)}, \hat{\boldsymbol{p}}_{k}^{(0)}, \mathbf{C}_{k}^{\boldsymbol{r}(0)}$, $\mathbf{C}_{k}^{\boldsymbol{p}(0)}$, measurement noise covariance $\mathbf{C}^{\boldsymbol{v}}, \mathbf{H}$ as defined in (5), multiplicative noise covariance $\mathbf{C}^{h}, \mathbf{F}$ and $\widetilde{\mathbf{F}}$ as defined in 23

Output: updated estimates $\hat{\boldsymbol{r}}_{k}^{\left(n_{k}\right)}, \hat{\boldsymbol{p}}_{k}^{\left(n_{k}\right)}$ and $\mathbf{C}_{k}^{r\left(n_{k}\right)}, \mathbf{C}_{k}^{\boldsymbol{p}\left(n_{k}\right)}$

For $i=1, \cdots, n_{k}$

$$
\begin{aligned}
& {\left[\begin{array}{lll}
\alpha & l_{1} & l_{2}
\end{array}\right]^{\mathrm{T}}=\boldsymbol{p}_{k}^{(i-1)}} \\
& \mathbf{S}=\left[\begin{array}{l}
\mathbf{S}_{\mathbf{1}} \\
\mathbf{S}_{\mathbf{2}}
\end{array}\right]=\left[\begin{array}{cc}
\cos \alpha & -\sin \alpha \\
\sin \alpha & \cos \alpha
\end{array}\right]\left[\begin{array}{cc}
l_{1} & 0 \\
0 & l_{2}
\end{array}\right] \\
& \mathbf{J}_{\mathbf{1}}=\left[\begin{array}{ccc}
-l_{1} \sin \alpha & \cos \alpha & 0 \\
-l_{2} \cos \alpha & 0 & -\sin \alpha
\end{array}\right] \\
& \mathbf{J}_{\mathbf{2}}=\left[\begin{array}{ccc}
l_{1} \cos \alpha & \sin \alpha & 0 \\
-l_{2} \sin \alpha & 0 & \cos \alpha
\end{array}\right] \\
& \mathbf{C}^{\mathrm{I}}=\mathbf{S C}^{h} \mathbf{S}^{\mathrm{T}} \\
& \mathbf{C}^{\mathrm{II}}=\left[\epsilon_{m n}\right]=\operatorname{tr}\left\{\mathbf{C}_{k}^{\boldsymbol{p}^{(i-1)}} \mathbf{J}_{n}^{\mathrm{T}} \mathbf{C}^{\boldsymbol{h}} \mathbf{J}_{m}\right\} \text { for } m, n=1,2 \\
& \mathbf{M}=\left[\begin{array}{c}
2 \mathbf{S}_{1} \mathbf{C}^{h} \mathbf{J}_{1} \\
2 \mathbf{S}_{2} \mathbf{C}^{h} \mathbf{J}_{2} \\
\mathbf{S}_{1} \mathbf{C}^{h} \mathbf{J}_{2}+\mathbf{S}_{\mathbf{2}} \mathbf{C}^{h} \mathbf{J}_{1}
\end{array}\right] \\
& \overline{\boldsymbol{y}}_{k}^{(i)}=\mathbf{H} \hat{\boldsymbol{r}}_{k}^{i-1} \\
& \mathbf{C}_{k}^{\boldsymbol{r} \boldsymbol{y}^{(i)}}=\mathbf{C}_{k}^{\boldsymbol{r ( i - 1 )}} \mathbf{H}^{\mathrm{T}} \\
& \mathbf{C}_{k}^{\boldsymbol{y}^{(i)}}=\mathbf{H C}_{k}^{\boldsymbol{r}(i-1)} \mathbf{H}^{\mathrm{T}}+\mathbf{C}^{\mathrm{I}}+\mathbf{C}^{\mathrm{II}}+\mathbf{C}^{\boldsymbol{v}} \\
& \boldsymbol{Y}_{k}^{(i)}=\mathbf{F}\left(\left(\boldsymbol{y}_{k}^{(i)}-\overline{\boldsymbol{y}}_{k}^{(i)}\right) \otimes\left(\boldsymbol{y}_{k}^{(i)}-\overline{\boldsymbol{y}}_{k}^{(i)}\right)\right) \\
& \overline{\boldsymbol{Y}}_{k}^{(i)}=\mathbf{F v e c t}\left\{\mathbf{C}_{k}^{\boldsymbol{y}^{(i)}}\right\} \\
& \mathbf{C}_{k}^{p \boldsymbol{Y}(i)}=\mathbf{C}_{k}^{\boldsymbol{p ( i - 1 )}} \mathbf{M}^{\mathrm{T}} \\
& \mathbf{C}_{k}^{\boldsymbol{Y}(i)}=\mathbf{F}\left(\mathbf{C}_{k}^{\boldsymbol{y}(i)} \otimes \mathbf{C}_{k}^{\boldsymbol{y}(i)}\right)(\mathbf{F}+\widetilde{\mathbf{F}})^{\mathrm{T}} \\
& \hat{\boldsymbol{r}}_{k}^{(i)}=\hat{\boldsymbol{r}}_{k}^{(i-1)}+\mathbf{C}_{k}^{\boldsymbol{r y} \boldsymbol{y}(i)}\left(\mathbf{C}_{k}^{\boldsymbol{y}(i)}\right)^{-1}\left(\boldsymbol{y}_{k}^{(i)}-\overline{\boldsymbol{y}}_{k}^{(i)}\right) \\
& \mathbf{C}_{k}^{r(i)}=\mathbf{C}_{k}^{\boldsymbol{r ( i - 1 )}}-\mathbf{C}_{k}^{\boldsymbol{r y} \boldsymbol{y}(i)}\left(\mathbf{C}_{k}^{\boldsymbol{y}(i)}\right)^{-1}\left(\mathbf{C}_{k}^{\boldsymbol{r y} \boldsymbol{y}(i)}\right)^{\mathrm{T}} \\
& \hat{\boldsymbol{p}}_{k}^{(i)}=\hat{\boldsymbol{p}}_{k}^{(i-1)}+\mathbf{C}_{k}^{\boldsymbol{p} \boldsymbol{Y}(i)}\left(\mathbf{C}_{k}^{\boldsymbol{Y}(i)}\right)^{-1}\left(\boldsymbol{Y}_{k}^{(i)}-\overline{\boldsymbol{Y}}_{k}^{(i)}\right) \\
& \mathbf{C}_{k}^{\boldsymbol{p}(i)}=\mathbf{C}_{k}^{\boldsymbol{p ( i - 1 )}}-\mathbf{C}_{k}^{\boldsymbol{p} \boldsymbol{Y ( i )}}\left(\mathbf{C}_{k}^{\boldsymbol{Y}(i)}\right)^{-1}\left(\mathbf{C}_{k}^{\boldsymbol{p} \boldsymbol{Y}(i)}\right)^{\mathrm{T}}
\end{aligned}
$$

End

1) Kinematic State Update: The kinematic state estimate is updated according to the Kalman filter update equations using the actual measurement $\boldsymbol{y}_{k}^{(i)}$, see $(\mathbf{C} 3)$,

$$
\begin{aligned}
\overline{\boldsymbol{y}}_{k}^{(i)} & =\mathbf{H} \hat{\boldsymbol{r}}_{k}^{(i-1)} \\
\hat{\boldsymbol{r}}_{k}^{(i)} & =\hat{\boldsymbol{r}}_{k}^{(i-1)}+\mathbf{C}_{k}^{\boldsymbol{r y} \boldsymbol{y}(i)}\left(\mathbf{C}_{k}^{\boldsymbol{y}(i)}\right)^{-1}\left(\boldsymbol{y}_{k}^{(i)}-\overline{\boldsymbol{y}}_{k}^{(i)}\right) \\
\mathbf{C}_{k}^{\boldsymbol{r}(i)} & =\mathbf{C}_{k}^{\boldsymbol{r ( i - 1 )}}-\mathbf{C}_{k}^{\boldsymbol{r y} \boldsymbol{y}(i)}\left(\mathbf{C}_{k}^{\boldsymbol{y}(i)}\right)^{-1}\left(\mathbf{C}_{k}^{\boldsymbol{r y} \boldsymbol{y}(i)}\right)^{\mathrm{T}}
\end{aligned}
$$

The challenge is to find compact closed-form approximations to the required moments, i.e., the covariance of the measurement $\mathbf{C}_{k}^{\boldsymbol{y}(i)}$ and the cross-correlation $\mathbf{C}_{k}^{r \boldsymbol{y}(i)}$ between the measurement and kinematic state.

The measurement equation (5) is linear in the kinematic state but nonlinear in the shape parameters due to the shape matrix $\mathbf{S}_{k}$. Linearizing $\mathbf{S}_{k} \boldsymbol{h}_{k}^{(i)}$ with respect to $\boldsymbol{p}_{k}$ at $\hat{\boldsymbol{p}}_{k}^{(i-1)}$ and keeping $\boldsymbol{h}_{k}^{(i)}$ as a random variable (C4) gives us

$$
\mathbf{S}_{k} \boldsymbol{h}_{k}^{(i)} \approx \underbrace{\hat{\mathbf{S}}_{k}^{(i-1)} \boldsymbol{h}_{k}^{(i)}}_{\mathrm{I}}+\underbrace{\left[\begin{array}{l}
\left(\boldsymbol{h}_{k}^{(i)}\right)^{\mathrm{T}} \widehat{\mathbf{J}}_{\mathbf{1}}^{(i-1)} \\
\left(\boldsymbol{h}_{k}^{(i)}\right)^{\mathrm{T}} \widehat{\mathbf{J}}_{\mathbf{2}}^{(i-1)}
\end{array}\right]\left(\boldsymbol{p}_{k}-\hat{\boldsymbol{p}}_{k}^{(i-1)}\right)}_{\text {II }}
$$


where $\hat{\bullet}_{k}^{(i-1)}$ denotes matrix $\bullet$ evaluated at the $(i-1)$-th shape estimate $\hat{\boldsymbol{p}}_{k}^{(i-1)}, \mathbf{J}_{\mathbf{1}}$ and $\mathbf{J}_{\mathbf{2}}$ are the Jacobian matrices of the first row and second row of $\mathbf{S}$, i.e.,

$$
\begin{aligned}
& \mathbf{J}_{\mathbf{1}}=\frac{\partial \mathbf{S}_{\mathbf{1}}}{\partial \boldsymbol{p}}=\left[\begin{array}{ccc}
-l_{1} \sin \alpha & \cos \alpha & 0 \\
-l_{2} \cos \alpha & 0 & -\sin \alpha
\end{array}\right], \\
& \mathbf{J}_{\mathbf{2}}=\frac{\partial \mathbf{S}_{\mathbf{2}}}{\partial \boldsymbol{p}}=\left[\begin{array}{ccc}
l_{1} \cos \alpha & \sin \alpha & 0 \\
-l_{2} \sin \alpha & 0 & \cos \alpha
\end{array}\right]
\end{aligned}
$$

with

$$
\mathbf{S}_{\mathbf{1}}=\left[\begin{array}{ll}
l_{1} \cos \alpha & -l_{2} \sin \alpha
\end{array}\right] \text { and } \mathbf{S}_{\mathbf{2}}=\left[\begin{array}{ll}
l_{1} \sin \alpha & l_{2} \cos \alpha
\end{array}\right] \text {. }
$$

Note that the terms I and II in (14) are uncorrelated. The covariance of $\mathbf{S}_{k} \boldsymbol{h}_{k}^{(i)}$ is approximated as the sum of $\mathbf{C}^{\mathrm{I}}$ and $\mathrm{C}^{\mathrm{II}}$, where

$$
\begin{aligned}
\mathbf{C}^{\mathrm{I}} & =\hat{\mathbf{S}}_{k}^{(i-1)} \mathbf{C}^{\boldsymbol{h}}\left(\hat{\mathbf{S}}_{k}^{(i-1)}\right)^{\mathrm{T}} \\
\underbrace{\left[\epsilon_{m n}\right]}_{\mathbf{C}^{\mathrm{II}}} & =\operatorname{tr}\left\{\mathbf{C}_{k}^{\boldsymbol{p}^{(i-1)}}\left(\widehat{\mathbf{J}}_{n k}^{(i-1)}\right)^{\mathrm{T}} \mathbf{C}^{\boldsymbol{h}}{\widehat{\mathbf{J}_{m k}}}^{(i-1)}\right\}
\end{aligned}
$$

for $m, n \in\{1,2\}$.

The derivation of (19) is shown in Appendix A-A The cross-covariance and covariance are

$$
\begin{aligned}
\mathbf{C}_{k}^{r \boldsymbol{y}^{(i)}} & =\mathbf{C}_{k}^{\boldsymbol{r}(i-1)} \mathbf{H}^{\mathrm{T}} \\
\mathbf{C}_{k}^{\boldsymbol{y}^{(i)}} & =\mathbf{H C}^{\boldsymbol{r ( i - 1 )}} \mathbf{H}^{\mathrm{T}}+\mathbf{C}^{\mathrm{I}}+\mathbf{C}^{\mathrm{II}}+\mathbf{C}^{\boldsymbol{v}} .
\end{aligned}
$$

2) Shape Update: A pseudo-measurement is constructed using the 2-fold Kronecker product (C3). For a twodimensional vector $\boldsymbol{y}=\left[\begin{array}{ll}y_{1} & y_{2}\end{array}\right]^{\mathrm{T}}$, its 2-fold Kronecker product $\otimes$ is defined as

$$
\boldsymbol{y} \otimes \boldsymbol{y}=\left[\begin{array}{llll}
y_{1}^{2} & y_{1} y_{2} & y_{2} y_{1} & y_{2}^{2}
\end{array}\right]^{\mathrm{T}} .
$$

Furthermore, each measurement is shifted by the expected measurement, and multiplied by a matrix

$$
\mathbf{F}=\left[\begin{array}{llll}
1 & 0 & 0 & 0 \\
0 & 0 & 0 & 1 \\
0 & 1 & 0 & 0
\end{array}\right] \text { or } \widetilde{\mathbf{F}}=\left[\begin{array}{llll}
1 & 0 & 0 & 0 \\
0 & 0 & 0 & 1 \\
0 & 0 & 1 & 0
\end{array}\right]
$$

to remove the duplicate element resulting from the 2-fold Kronecker product. All told, the pseudo-measurement is

$$
\boldsymbol{Y}_{k}^{(i)}=\mathbf{F}\left(\left(\boldsymbol{y}_{k}^{(i)}-\overline{\boldsymbol{y}}_{k}^{(i)}\right) \otimes\left(\boldsymbol{y}_{k}^{(i)}-\overline{\boldsymbol{y}}_{k}^{(i)}\right)\right)
$$

Note that 24) is an uncorrelated conversion (c.f., Theorem 3 in [36]), which means the pseudo-measurement is uncorrelated with the actual measurement.

The shape parameters are updated with the pseudomeasurement $\boldsymbol{Y}_{k}^{(i)}$ using the Kalman filter update formulas

$$
\begin{aligned}
\boldsymbol{p}_{k}^{(i)} & =\hat{\boldsymbol{p}}_{k}^{(i-1)}+\mathbf{C}_{k}^{p \boldsymbol{Y}(i)}\left(\mathbf{C}_{k}^{\boldsymbol{Y}(i)}\right)^{-1}\left(\boldsymbol{Y}_{k}^{(i)}-\overline{\boldsymbol{Y}}_{k}^{(i)}\right) \\
\mathbf{C}_{k}^{\boldsymbol{p}(i)} & =\mathbf{C}_{k}^{\boldsymbol{p}(i-1)}-\mathbf{C}_{k}^{\boldsymbol{p} \boldsymbol{Y}(i)}\left(\mathbf{C}_{k}^{\boldsymbol{Y}(i)}\right)^{-1}\left(\mathbf{C}_{k}^{\boldsymbol{p} \boldsymbol{Y}(i)}\right)^{\mathrm{T}}
\end{aligned}
$$

where $\overline{\boldsymbol{Y}}^{(i)}$ denotes the predicted pseudo-measurement, $\mathbf{C}_{k}^{\boldsymbol{Y}(i)}$ is the covariance of the pseudo-measurement, and $\mathbf{C}_{k}^{p Y(i)}$ is the cross-covariance between the pseudo-measurement and the shape parameters.
By constructing the pseudo-measurement in this way, the expected pseudo-measurement happens to consist of all centralized second moments of the actual measurements, which can be extracted directly from (21), see (C5). To show this, we introduce the vect-operator, which constructs a column vector from a matrix by stacking its column vectors. Given the covariance matrix of measurement $\mathbf{C}_{k}^{\boldsymbol{y}(i)}=\left[\begin{array}{ll}c_{11} & c_{12} \\ c_{12} & c_{22}\end{array}\right]$, a vect-operator gives us

$$
\operatorname{vect}\left\{\mathbf{C}_{k}^{\boldsymbol{y}(i)}\right\}=\left[\begin{array}{llll}
c_{11} & c_{12} & c_{12} & c_{22}
\end{array}\right]^{\mathrm{T}},
$$

which equals

$$
\mathrm{E}\left\{\left(\boldsymbol{y}_{k}^{(i)}-\overline{\boldsymbol{y}}_{k}^{(i)}\right) \otimes\left(\boldsymbol{y}_{k}^{(i)}-\overline{\boldsymbol{y}}_{k}^{(i)}\right)\right\} .
$$

The expected $i$-th pseudo-measurement is

$$
\overline{\boldsymbol{Y}}_{k}^{(i)}=\mathbf{F} \text { vect }\left\{\mathbf{C}_{k}^{\boldsymbol{y}(i)}\right\}
$$

The predicted pseudo-measurement covariance is

$$
\begin{aligned}
\mathbf{C}_{k}^{\boldsymbol{Y}(i)} & =\left[\begin{array}{ccc}
2 c_{11}^{2} & 2 c_{12}^{2} & 2 c_{11} c_{12} \\
2 c_{12}^{2} & 2 c_{22}^{2} & 2 c_{22} c_{12} \\
2 c_{11} c_{12} & 2 c_{22} c_{12} & c_{11} c_{22}+c_{12}^{2}
\end{array}\right] \\
& =\mathbf{F}\left(\mathbf{C}_{k}^{\boldsymbol{y}(i)} \otimes \mathbf{C}_{k}^{\boldsymbol{y}(i)}\right)(\mathbf{F}+\widetilde{\mathbf{F}})^{\mathrm{T}}
\end{aligned}
$$

Equation 30] is obtained using Isserlis's theorem [37] (see Appendix $\mathrm{A}-\mathrm{B}$. Equation 31 is a compact formulation of 30.

The cross-covariance between the pseudo-measurement and the shape parameters is approximated by linearization of 24. according to

$$
\mathbf{C}_{k}^{p \boldsymbol{Y}(i)}=\mathbf{C}_{k}^{\boldsymbol{p ( i - 1 )}}\left(\hat{\mathbf{M}}_{k}^{(i-1)}\right)^{\mathrm{T}}
$$

with

$$
\mathbf{M}=\left[\begin{array}{c}
2 \mathbf{S}_{1} \mathbf{C}^{h} \mathbf{J}_{1} \\
2 \mathbf{S}_{2} \mathbf{C}^{h} \mathbf{J}_{2} \\
\mathbf{S}_{1} \mathbf{C}^{h} \mathbf{J}_{2}+\mathbf{S}_{2} \mathbf{C}^{h} \mathbf{J}_{1}
\end{array}\right]
$$

The derivation of 33 is shown in the Appendix A-B1. The pseudo code of measurement update is given in Table $\mathrm{I}$.

\section{B. Time Update}

As the temporal evolution of both the kinematic state and the shape parameters follow a linear model, the time update can be performed with the standard Kalman filter time update formulas, i.e.,

$$
\begin{aligned}
\hat{\boldsymbol{r}}_{k+1}^{(0)} & =\mathbf{A}_{k}^{\boldsymbol{r}} \hat{\boldsymbol{r}}_{k}^{\left(n_{k}\right)}, \\
\mathbf{C}_{k+1}^{\boldsymbol{r}(0)} & =\mathbf{A}_{k}^{\boldsymbol{r}} \mathbf{C}_{k}^{\boldsymbol{r}\left(n_{k}\right)}\left(\mathbf{A}_{k}^{\boldsymbol{r}}\right)^{\mathrm{T}}+\mathbf{C}_{\boldsymbol{r}}^{\boldsymbol{w}} .
\end{aligned}
$$

and

$$
\begin{aligned}
\hat{\boldsymbol{p}}_{k+1}^{(0)} & =\mathbf{A}_{k}^{\boldsymbol{p}} \hat{\boldsymbol{p}}_{k}^{\left(n_{k}\right)} \\
\mathbf{C}_{k+1}^{\boldsymbol{p}(0)} & =\mathbf{A}_{k}^{\boldsymbol{p}} \mathbf{C}_{k}^{\boldsymbol{p}\left(n_{k}\right)}\left(\mathbf{A}_{k}^{\boldsymbol{p}}\right)^{\mathrm{T}}+\mathbf{C}_{\boldsymbol{p}}^{\boldsymbol{w}}
\end{aligned}
$$


No Measurement Noise, $\mathbf{C}^{v}=\mathbf{0}$

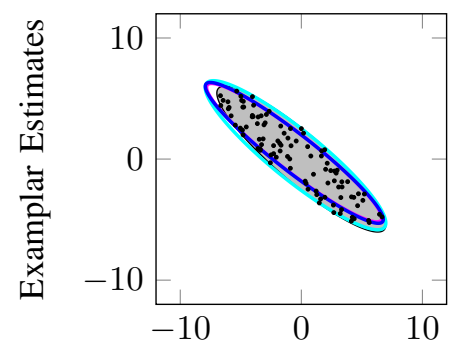

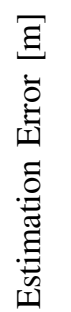

$x \rightarrow$

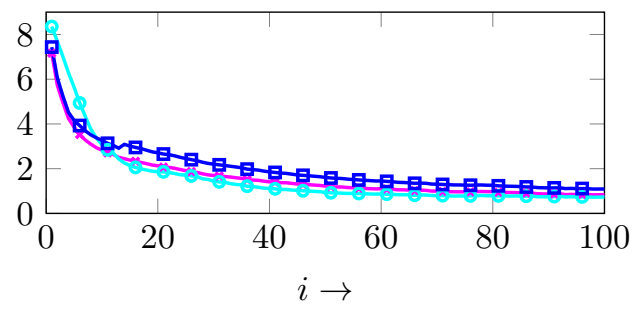

High Measurement Noise, $\mathbf{C}^{\boldsymbol{v}}=\operatorname{diag}\left[l_{1}, l_{2}\right]$
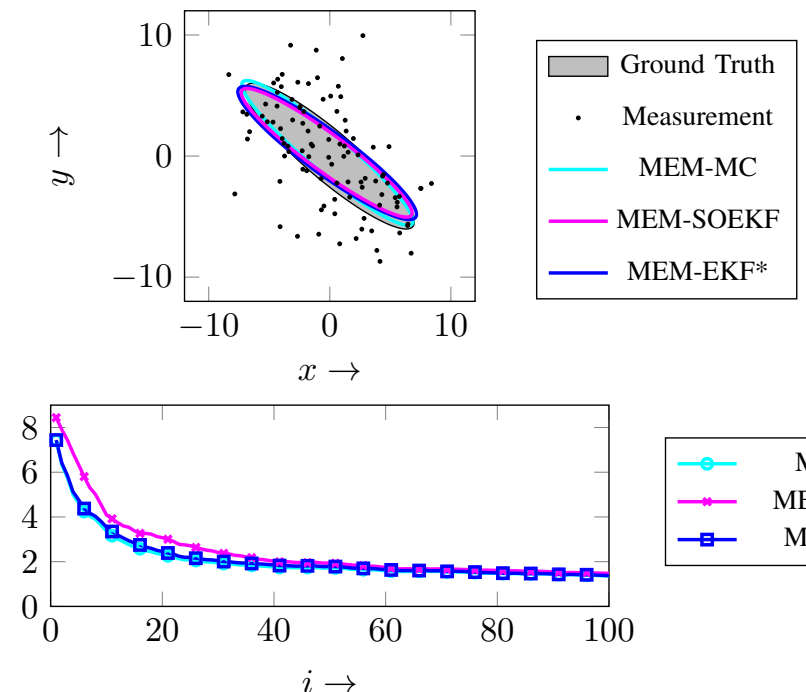

Fig. 3: Simulation with a stationary ellipse. The first row shows the ground truth, exemplar measurements, and the estimates after 100 measurement updates. The bottom row plots the root mean squared Gaussian Wasserstein distance averaged over 100 runs.

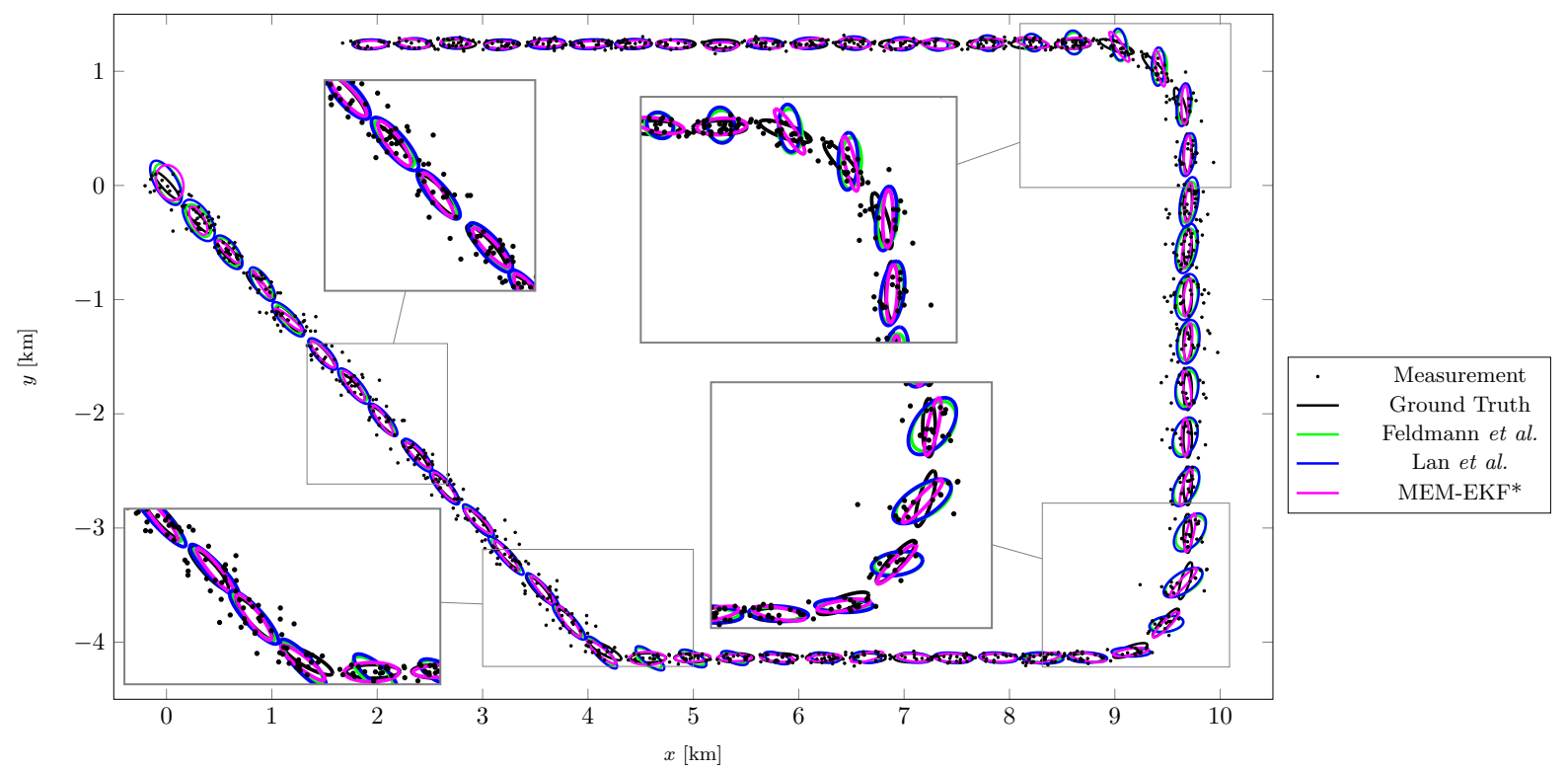

Fig. 4: The measurements, trajectory, and estimation results of a single example run.

\section{Evaluation}

In this section, we first evaluate the accuracy of the developed moment approximations. Then, the benefits of the developed shape tracker are demonstrated with respect to the random matrix approach in a simple extension dynamics. In the end, we integrate turn rate estimation and tested in a scenario in which object extent is coupled with its kinematics.

We assess location and extent errors simultaneously with a single score by means of the Gaussian Wasserstein distance [38] as proposed [39]. It is very important to note that orientation and axes-lengths errors are combined in a single scalar value.
The Gaussian Wasserstein distance compares two ellipses according to

$$
\begin{aligned}
d\left(\boldsymbol{\mu}_{1}, \boldsymbol{\Sigma}_{1}, \boldsymbol{\mu}_{2}, \boldsymbol{\Sigma}_{2}\right)^{2}=\left\|\boldsymbol{\mu}_{1}-\boldsymbol{\mu}_{2}\right\|^{2} \\
+\operatorname{tr}\left\{\boldsymbol{\Sigma}_{1}+\boldsymbol{\Sigma}_{2}-2 \sqrt{\sqrt{\boldsymbol{\Sigma}_{1}} \boldsymbol{\Sigma}_{2} \sqrt{\boldsymbol{\Sigma}_{1}}}\right\}
\end{aligned}
$$

where the ellipses are specified by their locations $\boldsymbol{\mu}_{1} \in \mathbb{R}^{2}$ and $\boldsymbol{\mu}_{2} \in \mathbb{R}^{2}$ and SPD shape matrices $\boldsymbol{\Sigma}_{1} \in \mathbb{R}^{2 \times 2}$ and $\boldsymbol{\Sigma}_{2} \in$ $\mathbb{R}^{2 \times 2}$. In this case, the first ellipse is the ground truth and the second one is the extended object tracking method estimate. Note that an SPD shape matrix is computed using $\hat{\mathbf{S}}_{k} \hat{\mathbf{S}}_{k}^{\mathrm{T}}$. 


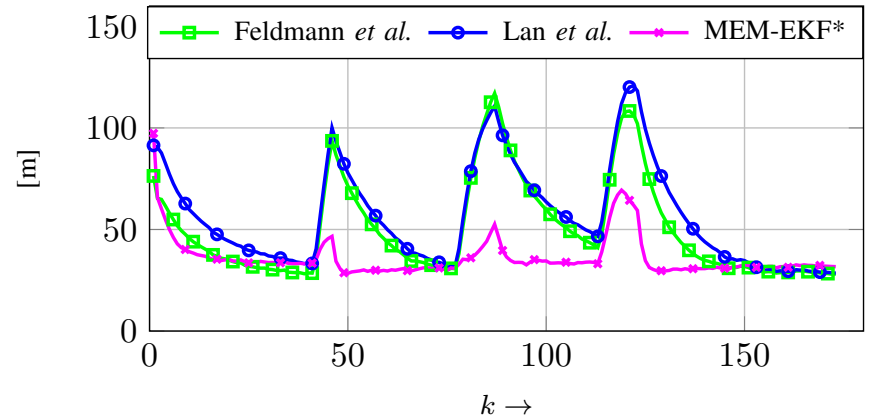

Fig. 5: Extent error based on the mean squared Gaussian Wasserstein distance.

\section{A. Evaluation of Moment Approximations}

First, we evaluate the quality of the proposed moment approximations for the kinematic state (12) and (13), and the shape parameters (25) and (26) compared to the Monte Carlo moment approximation and our Second-Order EKF [18], which requires the calculation of Hessian matrices. Both methods are computationally much more complex than the proposed tracker. As we focus on the moment approximations of the measurement update, we restrict ourselves to a scenario with a non-moving object. The considered object is located in the origin with semi-axes lengths 2 and 9 meters and it is counter-clockwise rotated $\frac{\pi}{3}$. The prior for the shape parameters is

$$
\begin{array}{ll}
\hat{\boldsymbol{r}}_{1}^{(0)}=\left[\begin{array}{ll}
1 & 1
\end{array}\right], & \mathbf{C}_{1}^{r(0)}=\operatorname{diag}\left[\begin{array}{ll}
1 & 1
\end{array}\right], \\
\hat{\boldsymbol{p}}_{1}^{(0)}=\left[\begin{array}{lll}
0 & 2 & 12
\end{array}\right], & \mathbf{C}_{1}^{p(0)}=\operatorname{diag}\left[\begin{array}{lll}
1 & 4 & 9
\end{array}\right]
\end{array}
$$

for all three methods. Two different measurement noise covariance matrices are evaluated and the simulation results are shown in Fig. 3 ,

As expected, the Monte Carlo moments approximation outperforms the analytic approaches in both low and high measurement noises scenarios. In case of high measurement noise, our moments approximation is almost as good as the Monte Carlo approximation unexpected. Fig. 3 shows that there are no significant visual differences between the methods. We can conclude that the derived moment approximations nearly matches the true exact moments in low and high noise scenarios.

\section{B. Comparison with Random Matrix Approaches using Con- stant Velocity Motion Models}

In the second simulation, we compare our algorithm with the two random matrix approaches by Feldmann et al. [12] and Lan et al. [23].

The considered scenario involves a target object with an unknown extent. However, only its orientation is changing over time, its semi-axes are fixed. With the MEM-EKF* we can assume a low system noise on the semi-axes lengths, and high system noise on the orientation. However, the random matrix approaches [12], [23] cannot model this scenario precisely (as there is only a single parameter for the extent uncertainty). For this reason, the MEM-EKF* is able to outperform the random

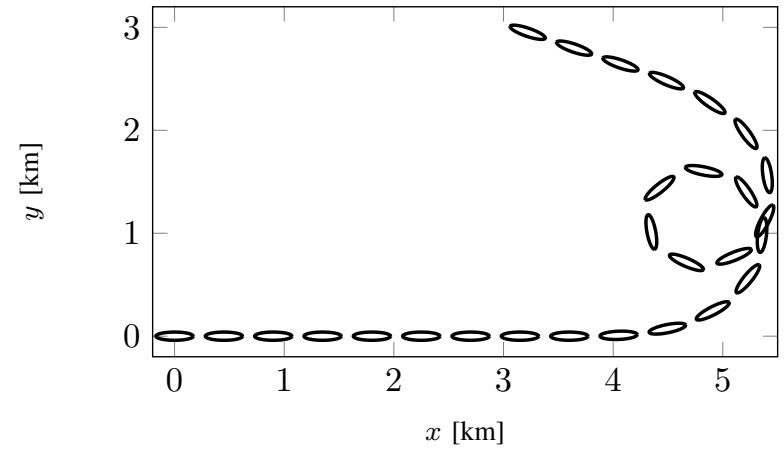

Fig. 6: True trajectory of variable turn simulation.

matrix approaches in this scenario. The true track is similar as in [12] and [23]. The extended object has diameters of $340 \mathrm{~m}$ and $80 \mathrm{~m}$. It starts at the coordinate origin and it moves with a constant speed of $50 \mathrm{~km} / \mathrm{h}$. At each time step, measurement sources are generated from a uniform distribution on the elliptical extent. The number of measurements per time scan is drawn from a Poisson distribution with mean 20 as in [23]. The variances of the measurement noise are $10000 \mathrm{~m}^{2}$ and $400 \mathrm{~m}^{2}$ for each dimension.

We set $\tau$ to 50 in the approach of Feldmann et al. ; $\delta$ to 40 in the approach of Lan et al. ; $v$ to 56 in both random matrix approaches. The extension transition matrix in Lan et al. 's approach is $\frac{1}{\delta_{k}} \mathbf{I}_{2}$. For our method, the prior of the shape variables is specified by the covariance matrix $\mathbf{C}_{0}^{p}=\operatorname{diag}\left[1,70^{2}, 70^{2}\right]$. The process noise covariance is set to $\mathbf{C}_{\boldsymbol{p}}^{\boldsymbol{w}}=\operatorname{diag}[0.1,1,1]$, and the transition matrix is $\mathbf{A}_{k}^{p}=\mathbf{I}_{3}$. The process noise covariance for the kinematic state is diag $[100,100,1,1]$ for all three estimators.

Measurements, trajectory, and estimation results of an example run are depicted in Fig. 4. Both random matrix trackers have worse results during turns. The extent error according to the Gaussian Wasserstein distance is depicted in Fig. 5 From Fig. 5 we can conclude that Feldmann et al. and Lan et al. perform similarly overall. This is expected as both random matrix methods are the same if no special dynamic model for the extend is used. For all three turns the proposed tracker has a lower error compared to both random matrix approaches due to the aforementioned assumption of (nearly) static semi-axis.

\section{Comparison with Random Matrix Approaches using Con- stant Turn Motion Models}

For a manoeuvring target, the object orientation is typically coupled with the turn rate [22]. Assuming that the size of the extended object is constant (plus noise), we can integrate the correlation between orientation and turn rate in the prediction of the MEM-EKF* according to

$$
\begin{aligned}
\hat{\boldsymbol{p}}_{k+1}^{(0)} & =\mathbf{B} \hat{\boldsymbol{r}}_{k}^{\left(n_{k}\right)}+\hat{\boldsymbol{p}}_{k}^{\left(n_{k}\right)}, \\
\mathbf{C}_{k+1}^{\boldsymbol{p}(0)} & =\mathbf{B} \mathbf{C}_{k}^{\boldsymbol{r}\left(n_{k}\right)} \mathbf{B}^{\mathrm{T}}+\mathbf{C}_{k}^{\boldsymbol{p}\left(n_{k}\right)}+\mathbf{C}_{\boldsymbol{p}}^{\boldsymbol{w}},
\end{aligned}
$$

where $\mathbf{B}=\left[\begin{array}{cc}\mathbf{0}_{1 \times 4} & T \\ \mathbf{0}_{2 \times 4} & \mathbf{0}_{2 \times 1}\end{array}\right]$ picks out turn rate $\hat{\Omega}_{k}^{\left(n_{k}\right)}$.

In this simulation, a target object that follows a variable turn-rate model is simulated, similar to [22]. The diameters of 


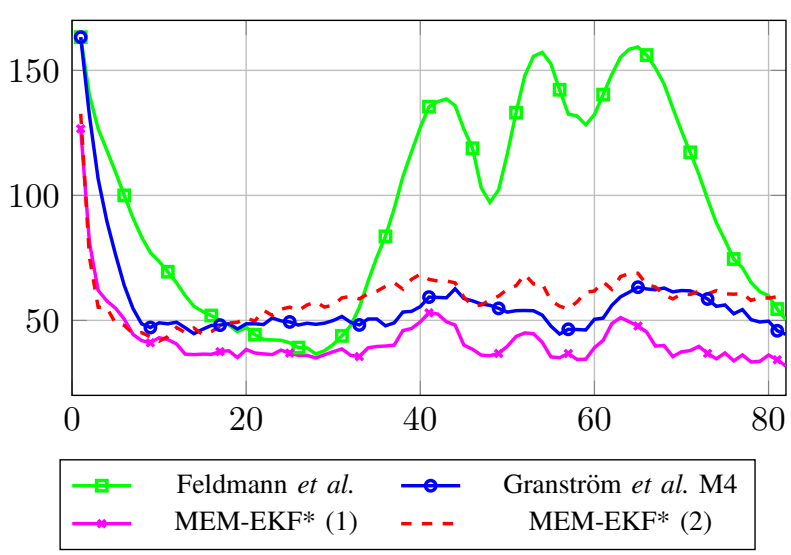

Fig. 7: Mean squared Gaussian Wasserstein distance for 100 runs.

the simulated object are $170 \mathrm{~m}$ and $40 \mathrm{~m}$ and the true track is shown in Fig. 6 In the first 25 time steps, the object moves with a constant velocity of $150 \mathrm{~m} / \mathrm{s}$. Afterwards, its turn rate increases from 0 to 20 degree per second in 20 time steps, and decreases to 0 in 20 time steps. The object evolves additional 5 time steps according to a constant velocity model in the end of the trajectory. The number of measurements for each time step is Poisson distributed with mean 20 and the measurement noise covariance is $\mathbf{C}^{v}=\operatorname{diag}[10000$ 400]. We compare the MEM-EKF* with the random matrix approach M4 proposed [22]. M4 approximates the density of the object extent with an inverse Wishart density by minimizing Kullback-Leibler divergence in the prediction step. As a baseline, we also include the random matrix approach from [12], which does not incorporate the turn-rate.

For all estimators, the object kinematics is modeled as a constant turn model and the prior is

$$
\begin{aligned}
\hat{\boldsymbol{r}}_{1}^{(0)} & =\left[\begin{array}{lllll}
100 & 100 & 100 & 20 & 0.001
\end{array}\right]^{\mathrm{T}} \\
\mathbf{C}_{1}^{\boldsymbol{r}(0)} & =\operatorname{diag}\left[\begin{array}{llll}
1600 \mathbf{I}_{2} & 16 \mathbf{I}_{2} & 0.001
\end{array}\right] .
\end{aligned}
$$

The parameters for the random matrix approaches are $v=56$, $\tau=5$, and $T=1 s$. The prior for our shape variables is

$$
\hat{\boldsymbol{p}}_{1}^{(0)}=\left[\begin{array}{lll}
\frac{\pi}{3} & 200 & 90
\end{array}\right]^{\mathrm{T}}, \quad \mathbf{C}_{1}^{\boldsymbol{p}(0)}=\operatorname{diag}\left[\begin{array}{ll}
0.2 & 360 \mathbf{I}_{2}
\end{array}\right] .
$$

The process noise covariace matrices for the location, velocity, and turn rate are

$$
\mathbf{C}_{\boldsymbol{r}}^{\boldsymbol{w}}=\operatorname{diag}\left[1000 \mathbf{I}_{2} \quad 100 \mathbf{I}_{2}\right], \quad \mathbf{C}_{\Omega}^{\boldsymbol{w}}=0.001 .
$$

The MEM-EKF* constrains the temporal evolution of the extent via process a suitable noise covariance for the shape parameters. In the same way as the simulation in Section IV-B, the process noise covariance matrix is tuned such that the semiaxes are only allowed to change slightly over time.

To demonstrate this effect, we choose two different process noise covariance matrices for our tracker and refer them as

$$
\begin{aligned}
& \text { MEM-EKF* (1) with } \quad \mathbf{C}_{p}^{\boldsymbol{w}}=\operatorname{diag}\left[.01 \quad \mathbf{I}_{2}\right] \\
& \text { MEM-EKF* (2) with } \quad \mathbf{C}_{\boldsymbol{p}}^{\boldsymbol{w}}=\operatorname{diag}\left[.1 \quad 40 \mathbf{I}_{2}\right]
\end{aligned}
$$

MEM-EKF* (1) has less process noise on the shape variables, i.e., only slight changes in the lengths of the semi-axes are possible. MEM-EKF* (2) has rather high process noise on the shape variables such that larger changes are possible.

The root mean squared Gaussian Wasserstein distance is given in Fig. 7 As expected, the tracking performance improves significantly with turn rate estimation and a constant turn motion model. MEM-EKF* (1) has the least estimation error, as size changes are constrained using small process noise correspondingly. However, note that, compared to random matrix approaches, MEM-EKF* involves more parameters. This is an advantage as well as a disadvantage. Its performance is sensitive to the choice of the parameters, e.g., the results of MEM-EKF* (2) are worse as the used parameters do not fit.

\section{CONClusion}

Extended object tracking is challenging - especially for the case of measurements that are scattered on the surface of the object. As the underlying estimation problem is highly nonlinear, closed-form solutions are rarely available.

In this work, we introduced a new closed-form tracker called MEM-EKF* for the orientation and axes-lengths of an elliptical extended object. For this purpose, an explicit measurement equation is formulated via a multiplicative error. A problem-tailored combination of analytic moment calculation and linearization techniques then allows to derive a Kalman filter-based measurement update. A major benefit of our method is that it provides an intuitive parameterization of an ellipse, which allows for directly modeling relevant motion models. Furthermore, the full joint covariance of the ellipse parameters is available. The closed-form formulas are compact, i.e., they are not significantly more complex than the standard Kalman filter formulas.

In the future, we will investigate extensions of the approach, e.g., for non-elliptical shapes or known extent [40] and it will be embedded into multi-object trackers such as the extended target PHD filter [21], [41].

\section{ACKNOWLEDGMENT}

This work was supported by the German Research Foundation (DFG) under grant BA 5160/1-1.

\section{APPENDIX A \\ DERIVATIONS FOR THE JOINT MOMENTS}

\section{A. Equation (19)}

As $\boldsymbol{h}$ is zero-mean 1 for $m, n=1,2$, we have

$$
\operatorname{cov}\left\{\left[\begin{array}{l}
\boldsymbol{h}^{\mathrm{T}} \widehat{\boldsymbol{J}_{1}} \\
\boldsymbol{h}^{\mathrm{T}} \widehat{\mathbf{J}_{\mathbf{2}}}
\end{array}\right] \boldsymbol{p},\left[\begin{array}{l}
\boldsymbol{h}^{\mathrm{T}} \widehat{\boldsymbol{J}_{1}} \\
\boldsymbol{h}^{\mathrm{T}} \widehat{\mathbf{J}_{\mathbf{2}}}
\end{array}\right] \boldsymbol{p}\right\}=\left[\epsilon_{m n}\right],
$$

\footnotetext{
${ }^{1}$ For the sake of compactness, we omit the measurement index $(i)$ and time index $k$
} 
with

$$
\begin{aligned}
& \epsilon_{m n}=\mathrm{E}\left\{\boldsymbol{h}^{\mathrm{T}} \widehat{\mathbf{J}_{\mathbf{m}}} \boldsymbol{p} \boldsymbol{p}^{\mathrm{T}}{\widehat{\mathbf{J}_{\mathbf{n}}}}^{\mathrm{T}} \boldsymbol{h}\right\}, \\
& =\mathrm{E}\left\{\operatorname{tr}\left\{\boldsymbol{h}^{\mathrm{T}} \widehat{\mathbf{J}_{\mathbf{m}}} \boldsymbol{p} \boldsymbol{p}^{\mathrm{T}}{\widehat{\mathbf{J}_{\mathbf{n}}}}^{\mathrm{T}} \boldsymbol{h}\right\}\right\}, \\
& =\mathrm{E}\left\{\operatorname{tr}\left\{\boldsymbol{p} \boldsymbol{p}^{\mathrm{T}}{\widehat{\mathbf{J}_{\mathbf{n}}}}^{\mathrm{T}} \boldsymbol{h} \boldsymbol{h}^{\mathrm{T}} \widehat{\mathbf{J}_{\mathbf{m}}}\right\}\right\} \text {, } \\
& =\operatorname{tr}\left\{\mathrm{E}\left\{\boldsymbol{p} \boldsymbol{p}^{\mathrm{T}}{\widehat{\mathbf{J}_{\mathbf{n}}}}^{\mathrm{T}} \boldsymbol{h} \boldsymbol{h}^{\mathrm{T}} \widehat{\mathbf{J}_{\mathbf{m}}}\right\}\right\} \text {, } \\
& =\operatorname{tr}\left\{\mathbf{C}^{p}{\widehat{\mathbf{J}_{\mathbf{n}}}}^{\mathrm{T}} \mathbf{C}^{h} \widehat{\mathbf{J}_{\mathbf{m}}}\right\} \text {. }
\end{aligned}
$$

Equation (48) follows from the fact that $\boldsymbol{h}^{\mathrm{T}} \widehat{\mathbf{J}_{\mathbf{m}}} \boldsymbol{p} \boldsymbol{p}^{\mathrm{T}}{\widehat{\mathbf{J}_{\mathbf{n}}}}^{\mathrm{T}} \boldsymbol{h}$ is $1 \times 1$. As trace is invariant under cyclical permutations, we have (49). Equation (50) follows from the property that trace is a linear operator and can commute with expectation. Equation (51) follows form the independence between $\boldsymbol{h}$ and $\boldsymbol{p}$.

\section{B. Pseudo-measurement Covariance}

To calculate the covariance of the, pseudo-measurement we need the fourth centralized moments of original measurement, which can be calculated using Isserlis's theorem [37] or Wick's theorem [42]. Given a measurement $\boldsymbol{y}=\left[\begin{array}{ll}y_{1} & y_{2}\end{array}\right]^{\mathrm{T}}$, the corresponding pseudo-measurement is

$$
\left[\begin{array}{l}
\boldsymbol{Y}_{1} \\
\boldsymbol{Y}_{2} \\
\boldsymbol{Y}_{3}
\end{array}\right]=\left[\begin{array}{c}
\left(y_{1}-\bar{y}_{1}\right)^{2} \\
\left(y_{2}-\bar{y}_{2}\right)^{2} \\
\left(y_{1}-\bar{y}_{1}\right)\left(y_{2}-\bar{y}_{2}\right)
\end{array}\right] .
$$

From Isserlis' theorem, we get

$$
\begin{aligned}
& \mathrm{E}\left\{\left(\boldsymbol{Y}_{1}\right)^{2}\right\}=3 c_{11}^{2} \\
& \mathrm{E}\left\{\left(\boldsymbol{Y}_{2}\right)^{2}\right\}=3 c_{22}^{2} \\
& \mathrm{E}\left\{\boldsymbol{Y}_{3} \boldsymbol{Y}_{1}\right\}=3 c_{11} c_{12}, \\
& \mathrm{E}\left\{\boldsymbol{Y}_{3} \boldsymbol{Y}_{2}\right\}=3 c_{22} c_{12} \\
& \mathrm{E}\left\{\left(\boldsymbol{Y}_{3}\right)^{2}\right\}=\mathrm{E}\left\{\boldsymbol{Y}_{1} \boldsymbol{Y}_{2}\right\}=c_{11} c_{22}+2 c_{12}^{2},
\end{aligned}
$$

where $c_{m n}$ denotes $\mathrm{E}\left\{\left(y_{m}-\bar{y}_{m}\right)\left(y_{n}-\bar{y}_{n}\right)\right\}$ for $m, n \in$ $\{1,2\}$. Based on the results above, the calculation of $m n$ th entry of pseudo-measurement covariance matrix simply follows

$$
\operatorname{cov}\left\{\boldsymbol{Y}_{m}, \boldsymbol{Y}_{n}\right\}=\mathrm{E}\left\{\boldsymbol{Y}_{m} \boldsymbol{Y}_{n}\right\}-\mathrm{E}\left\{\boldsymbol{Y}_{m}\right\} \mathrm{E}\left\{\boldsymbol{Y}_{n}\right\},
$$

for $m, n \in\{1,2,3\}$. After a few further calculations, we get covariance of pseudo-measurement as in (30).

1) Linearization of the Pseudo-measurement Equation: Let $\mathbf{S}_{1}$ and $\mathbf{S}_{2}$ denote the first and second row of matrix S. Similarly, $\mathbf{H}_{1}$ and $\mathbf{H}_{2}$ refer to the first and second row of $\mathbf{H}$. Accordingly, the pseudo-measurement equation 24) is rewritten as (time and measurement indices are omitted)

$$
g(\boldsymbol{r}, \boldsymbol{p})=\left[\begin{array}{c}
\left(\mathbf{H}_{\mathbf{1}} \boldsymbol{r}+\mathbf{S}_{\mathbf{1}} \boldsymbol{h}+v_{1}-\bar{y}_{1}\right)^{2} \\
\left(\mathbf{H}_{\mathbf{2}} \boldsymbol{r}+\mathbf{S}_{\mathbf{2}} \boldsymbol{h}+v_{2}-\bar{y}_{2}\right)^{2} \\
\left(\mathbf{H}_{\mathbf{1}} \boldsymbol{r}+\mathbf{S}_{\mathbf{1}} \boldsymbol{h}+v_{1}-\bar{y}_{1}\right)\left(\mathbf{H}_{\mathbf{2}} \boldsymbol{r}+\mathbf{S}_{\mathbf{2}} \boldsymbol{h}+v_{2}-\bar{y}_{2}\right)
\end{array}\right]
$$

The cross-covariance of the pseudo-measurement and shape parameters are approximated using

$$
\begin{aligned}
\mathbf{C}^{p \boldsymbol{Y}} & =\operatorname{cov}\left\{\frac{\partial g}{\left.\left.\partial \boldsymbol{p}\right|_{p=\hat{\boldsymbol{p}}}(\boldsymbol{p}-\hat{\boldsymbol{p}}), \quad \boldsymbol{p}\right\}}\right. \\
& =\mathbf{C}^{\boldsymbol{p}}(\underbrace{\mathrm{E}\left\{\left.\frac{\partial g}{\partial \boldsymbol{p}}\right|_{\boldsymbol{p}=\hat{\boldsymbol{p}}}\right\}}_{\widehat{\mathrm{M}}})^{\mathrm{T}}
\end{aligned}
$$

After applying the chain rule, $\frac{\partial g}{\partial \boldsymbol{p}}$ equals

$$
\left[\begin{array}{c}
2\left(\mathbf{H}_{1} \boldsymbol{r}+\mathbf{S}_{1} \boldsymbol{h}+v_{1}-\bar{y}_{1}\right) \boldsymbol{h}^{\mathrm{T}} \mathbf{J}_{\mathbf{1}} \\
2\left(\mathbf{H}_{\mathbf{2}} \boldsymbol{r}+\mathbf{S}_{\mathbf{2}} \boldsymbol{h}+v_{2}-\bar{y}_{2}\right) \boldsymbol{h}^{\mathrm{T}} \mathbf{J}_{\mathbf{2}} \\
\left(\mathbf{H}_{\mathbf{1}} \boldsymbol{r}+\mathbf{S}_{\mathbf{1}} \boldsymbol{h}+v_{1}-\bar{y}_{1}\right) \boldsymbol{h}^{\mathrm{T}} \mathbf{J}_{2}+\left(\mathbf{H}_{2} \boldsymbol{r}+\mathbf{S}_{\mathbf{2}} \boldsymbol{h}+v_{2}-\bar{y}_{2}\right) \boldsymbol{h}^{\mathrm{T}} \mathbf{J}_{1}
\end{array}\right]
$$

with $\mathbf{J}_{1}$ and $\mathbf{J}_{2}$ are given in (15) and (16). Evaluating the first row of (62) at $\hat{\boldsymbol{p}}$, we have

$$
2\left(\mathbf{H}_{\mathbf{1}} \boldsymbol{r}-\bar{y}_{1}\right) \boldsymbol{h}^{\mathrm{T}} \widehat{\mathbf{J}_{\mathbf{1}}}+2 \widehat{\mathbf{S}_{\mathbf{1}}} \boldsymbol{h} \boldsymbol{h}^{\mathrm{T}} \widehat{\mathbf{J}_{\mathbf{1}}}+2 v_{1} \boldsymbol{h}^{\mathrm{T}} \widehat{\mathbf{J}_{\mathbf{1}}}
$$

Taking the expectation of 63 gives us

$$
2 \widehat{\mathrm{S}_{1}} \mathbf{C}^{h} \widehat{\mathbf{J}_{1}}
$$

After a similar derivation for the second and third row of $(62)$, we get

$$
\widehat{M}=\left[\begin{array}{c}
2 \widehat{S_{1}} \mathbf{C}^{h} \widehat{J_{1}} \\
2 \widehat{\widehat{S}_{2}} \mathbf{C}^{h} \widehat{\mathbf{J}_{2}} \\
\widehat{\mathbf{S}_{1}} \mathbf{C}^{h} \widehat{\mathbf{J}_{2}}+\widehat{\mathbf{S}_{2}} \mathbf{C}^{h} \widehat{\mathbf{J}_{1}}
\end{array}\right]
$$

\section{REFERENCES}

[1] F. Kunz, D. Nuss, J. Wiest, H. Deusch, S. Reuter, F. Gritschneder, A. Scheel, M. Stübler, M. Bach, P. Hatzelmann, C. Wild, and K. Dietmayer, "Autonomous Driving at Ulm University: A Modular, Robust, and Sensor-Independent Fusion Approach," in IEEE Intelligent Vehicles Symposium (IV), Jun. 2015, pp. 666-673.

[2] T. Hirscher, A. Scheel, S. Reuter, and K. Dietmayer, "Multiple Extended Object Tracking using Gaussian Processes," in Proceedings of the 19th International Conference on Information Fusion (Fusion 2016), Jul. 2016, pp. 868-875.

[3] G. Vivone and P. Braca, "Joint Probabilistic Data Association Tracker for Extended Target Tracking Applied to X-Band Marine Radar Data," IEEE Journal of Oceanic Engineering, vol. 41, no. 4, pp. 1007-1019, Oct. 2016

[4] K. Granström, M. Baum, and S. Reuter, "Extended Object Tracking: Introduction, Overview and Applications," ISIF Journal of Advances in Information Fusion, vol. 12, no. 2, Dec. 2017.

[5] L. Mihaylova, A. Carmi, F. Septier, A. Gning, S. Pang, and S. Godsill, "Overview of Bayesian Sequential Monte Carlo Methods for Group and Extended Object Tracking," Digital Signal Processing, vol. 25, pp. 1-16, Feb. 2014.

[6] M. Baum and U. D. Hanebeck, "Shape Tracking of Extended Objects and Group Targets with Star-Convex RHMs," in Proceedings of the 14th International Conference on Information Fusion (Fusion 2011), Chicago, Illinois, USA, Jul. 2011.

[7] H. Kaulbersch, M. Baum, and P. Willett, "EM Approach for Tracking Star-Convex Extended Objects," in Proceedings of the 20th International Conference on Information Fusion (Fusion 2017), Xi' an, P.R. China, Jul. 2017.

[8] M. Baum and U. D. Hanebeck, "Extended Object Tracking with Random Hypersurface Models," IEEE Transactions on Aerospace and Electronic Systems, vol. 50, pp. 149-159, Jan. 2014.

[9] N. Wahlström and E. Özkan, "Extended Target Tracking Using Gaussian Processes," IEEE Transactions on Signal Processing, vol. 63, no. 16, pp. 4165-4178, Aug. 2015. 
[10] E. Özkan, N. Wahlström, and S. J. Godsill, "Rao-Blackwellised Particle Filter for Star-Convex Extended Target Tracking Models," in Proceedings of the 19th International Conference on Information Fusion (Fusion 2016), July 2016, pp. 1193-1199.

[11] W. Koch, "Bayesian Approach to Extended Object and Cluster Tracking using Random Matrices," IEEE Transactions on Aerospace and Electronic Systems, vol. 44, no. 3, pp. 1042-1059, Jul. 2008.

[12] M. Feldmann, D. Fränken, and W. Koch, "Tracking of Extended Objects and Group Targets using Random Matrices," IEEE Transactions on Signal Processing, vol. 59, no. 4, pp. 1409-1420, 2011.

[13] U. Orguner, K. Granstrom, and C. Lundquist, "Extended Target Tracking with a Cardinalized Probability Hypothesis Density Filter," in Proceedings of the 14th International Conference on Information Fusion (Fusion 2011), Chicago, Illinois, USA, Jul. 2011.

[14] J. Lan and X. Rong Li, "Tracking of Extended Object or Target Group Using Random Matrix - Part I: New Model and Approach," in Proceedings of the 15th International Conference on Information Fusion (Fusion 2012), Jul. 2012, pp. 2177-2184.

[15] R. Henriksen, "The Truncated Second-Order Nonlinear Filter Revisited," IEEE Transactions on Automatic Control, vol. 27, no. 1, pp. 247-251, 1982.

[16] M. Roth and F. Gustafsson, "An Efficient Implementation of the Second Order Extended Kalman Filter," in Proceedings of the 14th International Conference on Information Fusion (Fusion 2011), Chicago, Illinois, USA, July 2011.

[17] M. Baum, F. Faion, and U. D. Hanebeck, "Modeling the Target Extent with Multiplicative Noise," in Proceedings of the 15th International Conference on Information Fusion (Fusion 2012), Singapore, Jul. 2012.

[18] S. Yang and M. Baum, "Second-Order Extended Kalman Filter for Extended Object and Group Tracking," in Proceedings of the 19th International Conference on Information Fusion (Fusion 2016), Heidelberg, Germany, Jul. 2016

[19] — , "Extended Kalman Filter for Extended Object Tracking," in Proceedings of the 42nd IEEE International Conference on Acoustics, Speech, and Signal Processing (ICASSP 2017), New Orleans, USA, Mar 2017.

[20] F. Teich, S. Yang, and M. Baum, "GM-PHD filter for Multiple Extended Object Tracking based on the Multiplicative Error Shape Model and Network Flow Labeling," in Proceedings of the IEEE Intelligent Vehicles Symposium (IV 2017), Redondo Beach, CA, USA, Jun. 2017.

[21] S. Yang, F. Teich, and M. Baum, "Network Flow Labeling for Extended Target Tracking PHD Filters," IEEE Transactions on Industrial Informatics, pp. 1-1, 2019.

[22] K. Granström and U. Orguner, "A New Prediction for Extended Targets with Random Matrices," IEEE Transactions on Aerospace and Electrical Systems, 2013.

[23] J. Lan and X. R. Li, "Tracking of Extended Object or Target Group Using Random Matrix: New Model and Approach," IEEE Transactions on Aerospace and Electrical Systems, vol. 52, no. 6, pp. 2973-2988, Dec. 2016.

[24] J. Degerman, J. Wintenby, and D. Svensson, "Extended Target Tracking using Principal Components," in Proceedings of the 14th International Conference on Information Fusion (Fusion 2011), Chicago, Illinois, USA, Jul. 2011.

[25] B. Li, C. Mu, Y. Bai, J. Bi, and L. Wang, "Ellipse Fitting based Approach for Extended Object Tracking," Mathematical Problems in Engineering, vol. 2014, 2014.

[26] M. Baum, M. Feldmann, D. Fränken, U. D. Hanebeck, and W. Koch, "Extended Object and Group Tracking: A Comparison of Random Matrices and Random Hypersurface Models," in Proceedings of the IEEE ISIF Workshop on Sensor Data Fusion: Trends, Solutions, Applications (SDF 2010), Leipzig, Germany, Oct. 2010.

[27] G. Vivone, K. Granström, P. Braca, and P. Willett, "Multiple Sensor Measurement Updates for the Extended Target Tracking Random Matrix Model," IEEE Transactions on Aerospace and Electronic Systems, vol. 53, no. 5, pp. 2544-2558, 2017.

[28] D. Angelova, L. Mihaylova, N. Petrov, and A. Gning, "A Convolution Particle Filtering Approach for Tracking Elliptical Extended Objects," in Proceedings of the 16th International Conference on Information Fusion (Fusion 2013), Istanbul, Turkey, Jul. 2013, pp. 1542-1549.

[29] A. Swain and D. Clark, "The PHD Filter for Extended Target Tracking with Estimable Extent Shape Parameters of Varying Size," in 15th International Conference on Information Fusion (FUSION 2012), Singapore, Jul. 2012.

[30] F. Septier, S. K. Pang, S. Godsill, and A. Carmi, "Tracking of Coordinated Groups using Marginalised MCMC-based Particle Algorithm," in 2009 IEEE Aerospace conference, Big Sky, MT, USA, Mar. 2009.
[31] M. Wieneke and W. Koch, "A PMHT Approach for Extended Objects and Object Groups," IEEE Transactions on Aerospace and Electronic Systems, vol. 48, no. 3, pp. 2349-2370, Jul. 2012.

[32] P. Broßeit, B. Duraisamy, and J. Dickmann, "The Volcanormal Density for Radar-based Extended Target Tracking," in 2017 IEEE 20th International Conference on Intelligent Transportation Systems (ITSC), Oct 2017, pp. 1-6.

[33] K. Granström, C. Lundquist, and U. Orguner, "Tracking Rectangular and Elliptical Extended Targets Using Laser Measurements," in Proceedings of the 14th International Conference on Information Fusion (Fusion 2011), Chicago, Illinois, USA, Jul. 2011.

[34] K. Gilholm, S. Godsill, S. Maskell, and D. Salmond, "Poisson Models for Extended Target and Group Tracking," in SPIE: Signal and Data Processing of Small Targets, 2005.

[35] K. Gilholm and D. Salmond, "Spatial Distribution Model for Tracking Extended Objects," IEE Proceedings on Radar, Sonar and Navigation, vol. 152, no. 5, pp. 364-371, Oct. 2005.

[36] J. Lan and X. R. Li, "Nonlinear Estimation by LMMSE-Based Estimation With Optimized Uncorrelated Augmentation," IEEE Transactions on Signal Processing, vol. 63, no. 16, pp. 4270-4283, Aug 2015.

[37] L. Isserlis, "On A Formula For The Product-Moment Coefficient of Any Order of a Normal Frequency Distribution in Any Number of Variables," Biometrika, vol. 12, no. 1-2, pp. 134-139, 1918.

[38] C. R. Givens and R. M. Shortt, "A Class of Wasserstein Metrics for Probability Distributions," The Michigan Mathematical Journal, vol. 31, no. 2, pp. 231-240, 1984.

[39] S. Yang, M. Baum, and K. Granström, "Metrics for Performance Evaluation of Elliptic Extended Object Tracking Methods," in Proceedings of the 2016 IEEE International Conference on Multisensor Fusion and Integration for Intelligent Systems (MFI 2016), Baden-Baden, Germany, Sep. 2016.

[40] J. S. Fowdur, M. Baum, and F. Heymann, "Tracking Targets with Known Spatial Extent Using Experimental Marine Radar Data," in 22nd International Conference on Information Fusion (FUSION 2019), Ottawa, Canada, Jul. 2019.

[41] K. Granström and U. Orguner, "A PHD Filter for Tracking Multiple Extended Targets Using Random Matrices," IEEE Transactions on Signal Processing, vol. 60, no. 11, pp. 5657-5671, Nov. 2012.

[42] G. Wick, "The Evaluation of the Collision Matrix," Physical Review, vol. 80, no. 2, pp. 268-272, 1950. 